\title{
Dynamics of axially localized states in Taylor-Couette flows
}

\author{
Jose M. Lopez ${ }^{1,2, *}$ and Francisco Marques ${ }^{2, \dagger}$ \\ ${ }^{1}$ Institute of Fluid Mechanics, Friedrich-Alexander-Universität Erlangen-Nürnberg, D-91058 Erlangen, Germany \\ ${ }^{2}$ Departament de Física Aplicada, Universitat Politècnica de Catalunya, E-Barcelona 08034, Spain
}

(Received 23 February 2015; published 15 May 2015)

\begin{abstract}
We present numerical simulations of the flow confined in a wide gap Taylor-Couette system, with a rotating inner cylinder and variable length-to-gap aspect ratio. A complex experimental bifurcation scenario differing from the classical Ruelle-Takens route to chaos has been experimentally reported in this geometry. The wavy vortex flow becomes quasiperiodic due to an axisymmetric very low frequency mode. This mode plays a key role in the dynamics of the system, leading to the occurrence of chaos via a period-doubling scenario. Further increasing the rotation of the inner cylinder results in the appearance of a new flow pattern which is characterized by large amplitude oscillations localized in some of the vortex pairs. The purpose of this paper is to study numerically the dynamics of these axially localized states, paying special attention to the transition to chaos. Frequency analysis from time series simultaneously recorded at several points has been applied in order to identify the flow transitions taking place. It has been found that the very low frequency mode is essential to explain the behavior associated with the different transitions towards chaos including localized states.
\end{abstract}

DOI: 10.1103/PhysRevE.91.053011

PACS number(s): 47.20.Qr, 47.20.Ky, 47.11.Kb

\section{INTRODUCTION}

The formation of spatially localized patterns within a homogeneous background state is a regular feature in a wide variety of pattern forming systems (see [1] and references therein). In fluid dynamics localized states are particularly relevant in linearly stable flows, where the transition to turbulence occurs subcritically when the amplitude of the perturbation exceeds a certain parameter-dependent threshold. Flow patterns in which turbulent patches coexist with laminar flow emerge near the onset of transition and are believed to play a crucial role in organizing the complex dynamics of such a transition. Examples of these localized states are found in canonical wall-bounded shear flows such as pipe flow [2-5] or plane Couette flow [6-9].

Spatially localized structures have also been observed in Taylor-Couette flow in both subcritical and supercritical scenarios. Nevertheless, in contrast to the above-mentioned canonical flows, localization does not necessarily involve coexistence of laminar and turbulent flow, but sometimes manifests itself in the appearance of spatially confined regions in which some feature of the global background state is locally modified. An example of this phenomenon was reported by Heisse et al. [10], who using a combined experimental and numerical approach found the existence of spiral vortices with strongly localized amplitude near the end plates in the case of centrifugally stable counter-rotating cylinders. Also in this regime of Taylor-Couette flow, Abshagen et al. [11] showed the emergence of steady and axisymmetric vortices localized near the inner cylinder. These vortices appear smoothly in the basic state, resulting in multiplicity of localized states which differ among themselves in the total number of vortices. The presence of a large-scale circulation due to the existence of the end walls seems to play a key role in the formation of these vortices. They could be related to a homoclinic snaking

\footnotetext{
*jose.lopez@fau.de

†francisco.marques@upc.edu
}

branch. More recently, axially localized states have been experimentally reported in the centrifugally unstable regime, in the case of a rotating inner cylinder and stationary outer cylinder [12]. These pre-turbulent states occur for sufficiently large values of the inner cylinder Reynolds number Re, in systems in which the ratio between the length-to-gap aspect ratio $\Gamma$ and the number of vortices $N$ is restricted to the range $0.85 \simeq \Gamma / N \simeq 0.95$. They are characterized by the existence of large amplitude oscillations localized in some of the outgoing jets of the bulk vortices. In all of these studies, despite the differences between the resulting states or the regime of Taylor-Couette flow considered, the phenomenon of localization results from the finite nature of the experimental facilities, because of the interaction between the bulk flow and the Ekman vortices generated at the end plates. The competition between states with a different number of vortices (Eckhaus instability), modulated by the presence of end walls, also plays a role in the localization. Thus, simulations in axially periodic systems cannot capture this dynamics. In [11] the background state over which localization takes place is a boundary-driven large-scale circulation, which is a major difference with the laminar states in other linearly stable flows, whereas in [10] and [12], localization occurs over a global state, in which the oscillation amplitude of some vortices differ from that in the background state. In the former, this global background state consists of spirals propagating from mid-height towards the ends and vice versa, and in the latter, it is the bifurcated state (wavy vortex flow) resulting after the Taylor-vortex flow (TVF) becomes unstable. This bifurcated state was termed the "small jet" (SJ) state $[13,14]$.

The bifurcation scenario exhibited by the fluid in the experimental setup utilized in [12] (wide gap $r_{i} / r_{0}=0.5$ and moderate-large $\Gamma$ ) shows some very interesting dynamical features. A variety of states and transitions between them have been experimentally reported in $[12,14,15]$. These occur for all TVF in the $\Gamma / N$ range mentioned before, as long as the number of vortices is $N \geqslant 8$. A complex transition to chaos takes place, differing from the classical Ruelle-Takens scenario often associated with Taylor-Couette flows [16]. When Re 
is increased above a certain $\mathrm{Re}_{\mathrm{VLF}}$ from the small jet $\mathrm{SJ}$ state, the flow becomes quasiperiodic due to the emergence of a very low frequency mode. However, in contrast to other modulated wavy flows (MWVF) [17,18], this mode is axisymmetric $(n=0)$. Further examples of a nonlinearly driven axisymmetric mode have been reported in other regimes of Taylor-Couette flows $[19,20]$. The very low frequency mode (VLF), as was termed in [14], plays an essential role in the dynamics of the flow, leading to the occurrence of chaos through a period-doubling route of two tori [15]. This chaotic regime vanishes with further increase in $\mathrm{Re}$, giving way to the flow regime characterized by the existence of global states with axially localized large amplitude oscillations [12]. In this regime, localization may result in a large multiplicity of states which coexist in a wide range of the parameter space $(\Gamma, \mathrm{Re})$.

An idealized finite Taylor-Couette system is invariant under rotations around the axis, and reflection about the equatorial plane. However, these symmetries are only approximately fulfilled in any real system, where the presence of noise, imperfections, and/or other phenomena results in symmetry breaking. Therefore, when complex dynamical behavior is observed in such systems, one may wonder whether this is an intrinsic feature of the system or, conversely, if it is driven by external sources such as noise or imperfections. There are several examples in fluid problems where imperfections and/or noise alter the dynamics of idealized systems, leading to additional bifurcations [21-25]. There also exist situations in which the presence of noise may entirely change the dynamics. An example is when a succession of rapid bifurcations occurs in a narrow parameter range. Here, the existence of noise usually induces a chaotic drift between the different states, resulting in a sudden transition to chaos [26,27]. Another example is found in systems that are particularly sensitive to perturbations. These excite the unstable states, giving rise to long transients that eventually decay unless they are sustained by ongoing noise $[28,29]$.

The aim of this paper is to extend the available information on the axially localized state regime (ALS) and very low frequency mode (VLF) by means of a detailed numerical study of the parameter space in which these states occur. In numerical simulations we can enforce the symmetries of the system and reduce the noise to machine accuracy, so that the intrinsic or extrinsic nature of the observed states and dynamics can be elucidated. Due to the multiplicity of states, the dynamics is very complex and numerous sequences of previously unreported transitions have been found when the control parameters Re and $\Gamma$ are varied. The VLF occurs in a wide range of the parameter space and its interaction with the localized states ALS appears to be crucial in most transitions, either between different ALS or to chaos. The rest of this paper is structured as follows. The governing equations and numerical method used to solve them are discussed in Sec. II. In Sec. III we briefly describe the sequence of states preceding the ALS regime, paying especial attention to the VLF and the transition to ALS. The family of ALS found in this particular case is shown in Sec. IV A. Its main features are enumerated and compared with the experimental results. In Sec. IV B, the sequences of states exhibited by the fluid in the transition to chaos when $\operatorname{Re}$ and $\Gamma$ are varied are illustrated. Finally, the main remarks are collected in Sec. V.

\section{GOVERNING EQUATIONS AND NUMERICAL METHOD}

\section{A. Description of the system}

We consider an incompressible fluid of kinematic viscosity $v$ confined between two concentric cylinders of length $h$ and inner and outer radii $r_{i}$ and $r_{o}$. The inner cylinder rotates at constant angular speed $\Omega$, whereas the top and bottom end walls and outer cylinder remain at rest. The system is nondimensionalized using the gap width, $d=r_{o}-r_{i}$, as the length scale and the viscous time $d^{2} / v$ as the time scale. The radius ratio is $r_{i} / r_{o}=0.5$. The inner cylinder Reynolds number Re and the aspect ratio $\Gamma$ are the control parameters of the system. The flow is governed by the incompressible Navier-Stokes equations,

$$
\partial_{t} \mathbf{v}+(\mathbf{v} \cdot \nabla) \mathbf{v}=-\nabla p+\Delta \mathbf{v}, \quad \nabla \cdot \mathbf{v}=0,
$$

where $\mathbf{v}=(u, v, w)$ denotes the nondimensional velocity field in cylindrical coordinates $(r, \theta, z)$. The no-slip boundary conditions are

$$
\begin{aligned}
\mathbf{v}\left(r_{i}, \theta, z, t\right) & =(0, \operatorname{Re}, 0), \\
\mathbf{v}\left(r_{o}, \theta, z, t\right) & =(0,0,0), \\
\mathbf{v}(r, \theta, \pm \Gamma / 2, t) & =(0,0,0) .
\end{aligned}
$$

For axisymmetric solutions the meridional components of velocity can be written in terms of the Stokes stream function $\psi, u=-1 / r \partial \psi / \partial z$ and $w=1 / r \partial \psi / \partial r$. The streamlines on a meridional $(\theta$-constant) plane are $\psi$-constant curves.

The governing equations and boundary conditions are invariant under arbitrary rotations $R_{\phi}$ about the axis, and the reflection $K_{z}$ about the equatorial plane $z=0$. The actions of these symmetries on the velocity field are

$$
\begin{gathered}
R_{\phi} \mathbf{v}(r, \theta, z, t)=\mathbf{v}(r, \theta+\phi, z, t), \\
K_{z} \mathbf{v}(r, \theta, z, t)=(u, v,-w)(r, \theta,-z, t) .
\end{gathered}
$$

Together, they generate the symmetry group $\mathcal{G}=\mathrm{SO}(2) \times Z_{2}$ of the system.

\section{B. Numerical formulation and methodology}

The governing equations (1) have been solved using a second-order time-splitting method [30]. The spatial discretization is via a Galerkin-Fourier expansion in $\theta$ and Chebyshev collocation in $x=2 r-r_{i}-r_{o}$ and $y=2 z / \Gamma$, of the form,

$$
F(r, \theta, z)=\sum_{l=0}^{L} \sum_{n=0}^{N} \sum_{m=-M}^{M} a_{l, n, m} T_{l}(x) T_{n}(y) e^{i m \theta},
$$

where $F$ represents the three velocity components and pressure. For each Fourier mode in (5), the corresponding Helmholtz and Poisson equations are solved efficiently by using a complete diagonalization of the operators in both the radial and axial directions. In order to decouple the Helmholtz equations for $u$ and $v$, we have used the combinations $u_{+}=$ $u+i v$ and $u_{-}=u-i v$ [31].

The idealized boundary conditions (2) are discontinuous at the junction where the stationary end walls meet the rotating cylinder, $\left(r=r_{i}, z= \pm \Gamma / 2\right)$. In a physical experiment 
(a)

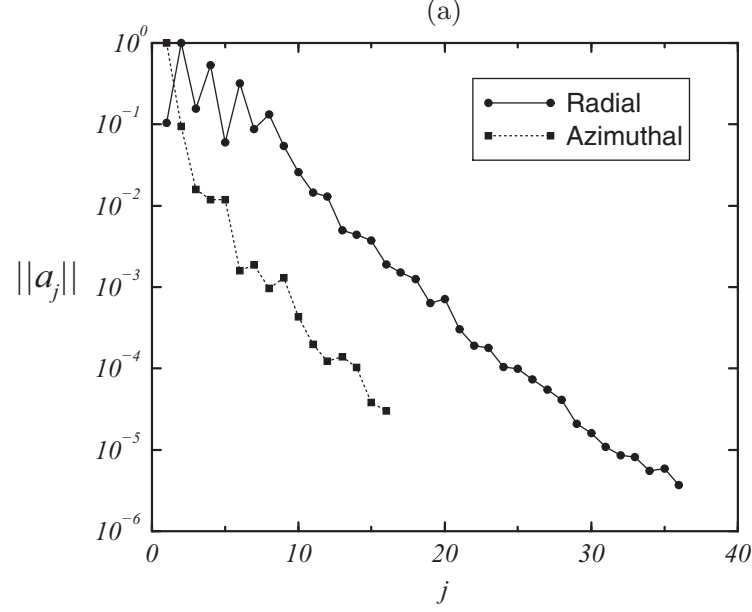

(b)

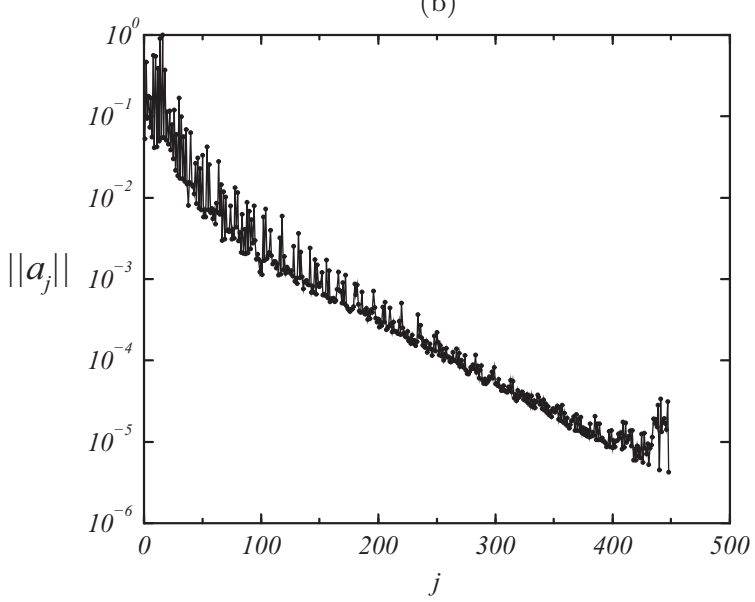

FIG. 1. Convergence of the spectral coefficients (5) of the axial velocity using the infinity norm. The flow corresponds to a weakly chaotic state found at $\operatorname{Re}=1300$ and $\Gamma=9$, computed with $L=36$ Chebyshev radial points, $M=16$ Fourier modes, and $N=448$ Chebyshev axial points. (a) Convergence in $r$ and $\theta$ and (b) in $z$.

there are small but finite gaps at these junctions where the azimuthal velocity adjusts rapidly to zero. For an accurate use of spectral techniques, a regularization of these discontinuities is implemented of the form,

$$
v(r, \theta, \pm \Gamma / 2, t)=\operatorname{Re} \exp \left(\frac{r_{i}-r}{\epsilon}\right),
$$

where $\epsilon$ is a small parameter that mimics the small physical gap (we have used $\epsilon=0.01$ ). The use of $\epsilon \neq 0$ regularizes the otherwise discontinuous boundary conditions; see [32] for further details on the use of this technique in spectral codes.

A MPI parallelization of the code has been accomplished by distributing the total number of azimuthal nodes $n n=2 M$ among the number of processors used in each simulation. Consequently, the maximum number of parallel tasks to be performed must be $\leqslant n n$. This arrangement is possible because the system of equations resulting from the discretization is independent for each Fourier mode. Further details about the parallelization strategy can be found in Ref. [33].

The spectral solver used here has been extensively tested in enclosed annular domains $[34,35]$ and it is based on a previous scheme used in a wide variety of flows in enclosed cylinders [36-40]. We have checked the spectral convergence of the code using the infinity norm of the spectral coefficients of the computed solutions, defined as $\left\|a_{l}\right\|_{\infty}=\max _{n, m}\left|a_{l, n, m}\right|$ for the radial direction, and analogously for the axial and azimuthal directions. Figure 1 shows $\left\|a_{j}\right\|_{\infty}$, with $j=l, n, m$, of the axial velocity $w$ for a weakly chaotic state taking place at $\operatorname{Re}=1300$ and $\Gamma=9$. This solution has been computed with $L=36$ and $N=448$ Chebyshev points in $r$ and $z$, and $M=16$ Fourier modes in $\theta$, ensuring that the trailing coefficients of the spectral expansion (5) are at least four orders of magnitude smaller than the leading coefficients. Time steps as small as $\delta t=5 \times 10^{-6}$ have been required for numerical stability and accuracy of the second-order temporal scheme.

Time series of the axial velocity at three different points, $w_{1}(r=1.2, \theta=0, z=\Gamma / 4), w_{2}(r=1.2, \theta=0, z=-\Gamma / 4)$, and $w_{3}(r=1.2, \theta=0, z=0)$, are simultaneously recorded. A subsequent spectral analysis of these data via fast Fourier transform, together with phase portraits and Poincare sections are used as tools to identify the different transitions that take place as the control parameters are varied (see [41] for a description of these methods).

\section{FLOW PATTERNS AND TRANSITIONS TOWARDS THE ALS REGIME}

\section{A. TVF}

There exist a variety of steady TVF, differing in the number of vortices $N$ present in the fluid domain, which coexist for the same values of $\Gamma$ and Re. They are very sensitive to the initial conditions of the system and thus depend on the path followed in parameter space. The choice of a certain TVF as the initial state determines in many cases the sequence of transitions exhibited by the fluid as $\mathrm{Re}$ is increased. However, the bifurcation scenario described along this paper is very robust, taking place in the same region as a normalized parameter space $(\Gamma / N, \operatorname{Re})$, provided that $N \geqslant 8$ (see Fig. 2 in [15] and Figs. 5 and 7 in [12]). In this study we focus on a TVF with $N=10$ vortices, which allows us to find a significant number of axially localized states in moderate aspect ratio systems $(8.5<\Gamma<9.5)$ at an affordable computational cost. In addition, this complements the experimental work of [12], in which axially localized states were reported for the cases of $N=8$ and $N=12$ vortices. Figure 2 illustrates the streamlines (a) and angular momentum (b) of this state. Five outgoing jets emerge from the inner cylinder due to the centrifugal instability. The two cells at the cylinder ends are larger than the others due to the Ekman layers resulting from the interaction between fluid and end walls. The kinetic energy of the fluid is concentrated in the boundary layer at the inner cylinder and in the outgoing jets, which are thinner and more intense than the return ingoing jets.

\section{B. Small and large jet states}

The axisymmetric five-jet TVF state loses stability at $\operatorname{Re} \approx 425$ via a Hopf bifurcation to a rotating wave with azimuthal wave number $m=1$. Figure 3 shows contours of 
(a)

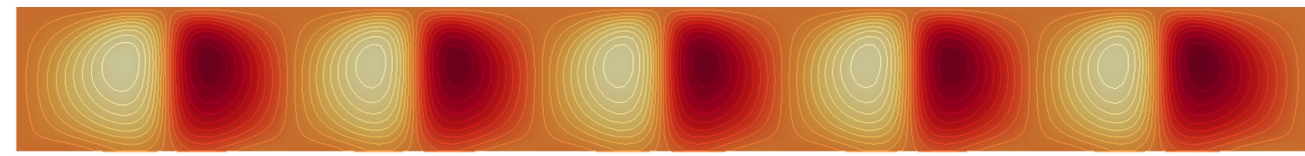

(b)

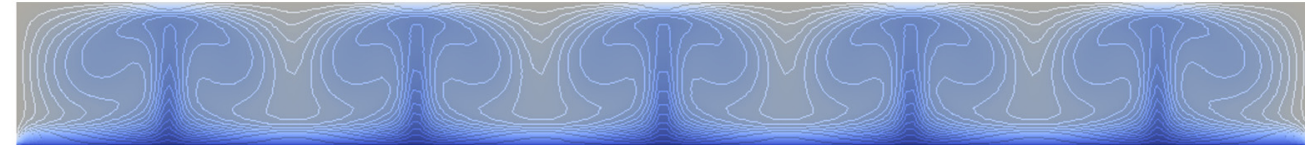

FIG. 2. (Color online) Axisymmetric five-jet (10 vortices) TVF state at $\operatorname{Re}=400$ and $\Gamma=9$ on a meridional plane $(r, z) \in[1,2] \times$ $[-\Gamma / 2, \Gamma / 2]$. (a) Streamlines. There are 10 positive (red/dark gray) and negative (yellow/light gray) $\psi$ contours in [-28.45,28.45]. (b) Contours of angular momentum $r v$. There are 20 contours in $[0,400]$.

angular momentum of the resulting state at $\mathrm{Re}=700$ and $\Gamma=9$ on cylindrical $(\theta, z)(\mathrm{a})$, and meridional $(r, z)(\mathrm{b})$ sections of the apparatus. The $m=1$ oscillations mainly occur at the outgoing jets, while the ingoing jets remain in the same axial place for all values of $\theta$. The exception are the two outgoing jets near the end walls which also remain stationary. A phase difference of 180 degrees between oscillations in contiguous outgoing jets can be seen from Fig. 3(a). These features are in full agreement with experimental observations [14] as well as with the numerical work by Jones in infinite long cylinders [13]. Figure 3(c) shows the evolution of the axial velocity $w$ over the line $r=1.8, \theta=\pi, z \in[-\Gamma, \Gamma]$, recorded at 20 different time steps and overlapped on the same plot. The oscillations of the outgoing jets are reflected in the thicker lines located around the outgoing jets in the bulk. As a result of the oscillations the axial velocity in these jets is larger than that in the jets at the Ekman vortices. Since the outgoing jets are thinner than the ingoing ones, the terminology small-jet state SJ and small jet instability has been typically used in the literature to refer to this state. Both the $\mathrm{SO}(2)$ rotational symmetry and the $K_{z}$ reflection symmetry $(z \rightarrow-z)$ of the TVF are broken in the Hopf bifurcation to the SJ, but this state still retains a discrete spatial $Z_{2}$ symmetry, the composition of a half-turn (a rotation of $\pi$ around the cylinder axis) and the $K_{z}$ reflection. This symmetry, which can be observed in Fig. 3(a), is the central reflection (also called the inversion $\mathcal{I}$ or shift-and-reflect symmetry) with respect to the center of the cylindrical domain $(r=0$ and $z=0)$. The spatial $\mathrm{SO}(2)$ rotational symmetry of the TVF becomes a spatiotemporal symmetry of the rotating wave: A rotation of angle $\alpha$ is equivalent to a time evolution of $t=\alpha / f_{p}$, where $f_{p}$ is the azimuthal drift frequency of the SJ. The value of $f_{p}$ varies between $44 \%-48 \%$ of the rotation frequency of the inner cylinder as Re is increased.

There exists another global wavy vortex flow which coexists with the SJ in a wide region of the parameter space (for $\operatorname{Re}>700$ ), and is characterized by the large amplitude of its oscillations. For this reason it is referred to as large jet $\mathrm{LJ}$, although most authors call it just wavy vortex flow (WVF) $[42,43]$. In order to compare the SJ and LJ states, Fig. 4 illustrates the LJ state at $\mathrm{Re}=720$ and $\Gamma=9.25$ similarly to Fig. 3. The main oscillations happen again in the outgoing jets, showing a substantial increase in the amplitude of the oscillations with respect to that in the SJ. Nevertheless, in contrast to SJ, the outgoing jets oscillate in phase. Oscillations of the ingoing jets can be discerned in this flow pattern. The amplitude of these oscillations is much lower than that in the outgoing jets and they occur in antiphase with the oscillations (a)

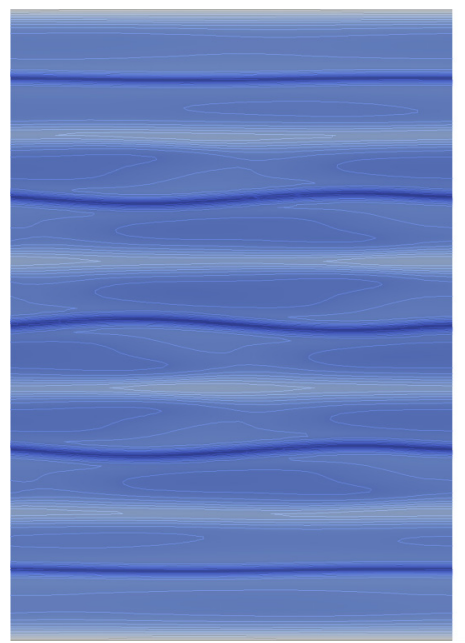

(b)

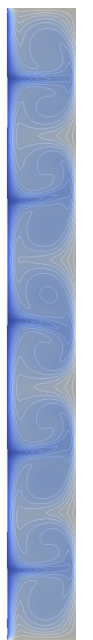

(c)

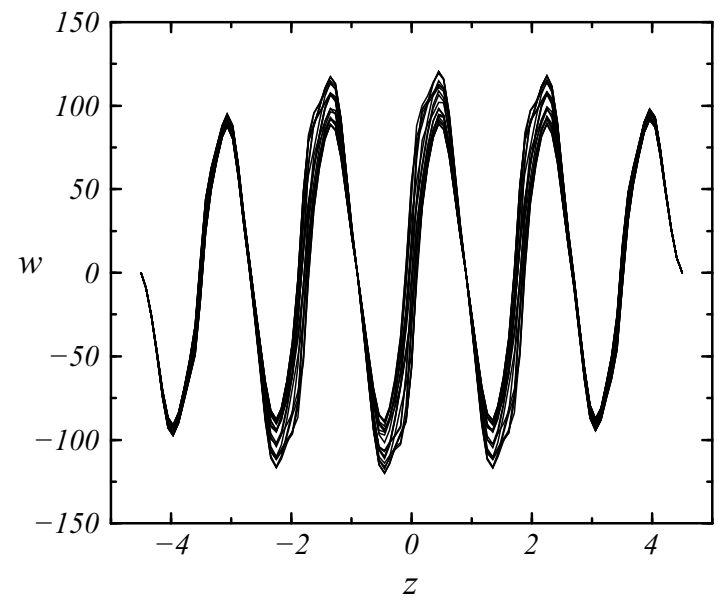

FIG. 3. (Color online) Small jet mode at $\operatorname{Re}=700$ and $\Gamma=9$. Contours of angular momentum $r v$, (a) on a cylindrical surface $(\theta, z) \in$ $[0,2 \pi] \times[-\Gamma / 2, \Gamma / 2]$ at $r=1.5$ and (b) on a meridional plane $(r, z) \in[1,2] \times[-\Gamma / 2, \Gamma / 2]$ at $\theta=\pi$. (c) Overlapped axial velocity profiles $w$ over the line $r=1.8, \theta=\pi, z \in[-\Gamma / 2, \Gamma / 2]$, corresponding to 20 different time steps. 
(a)

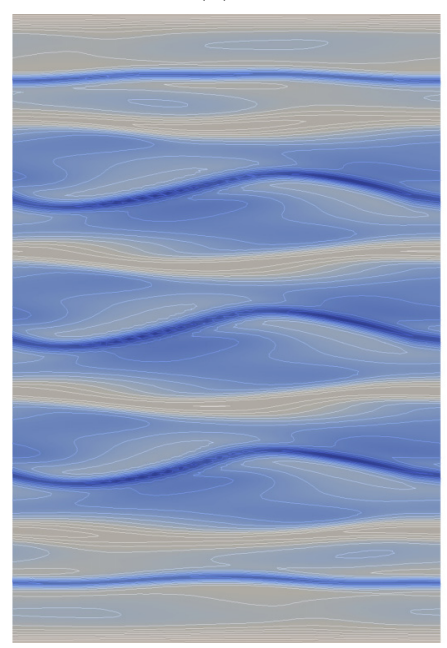

(b)

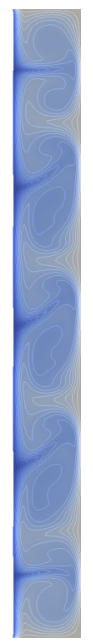

(c)

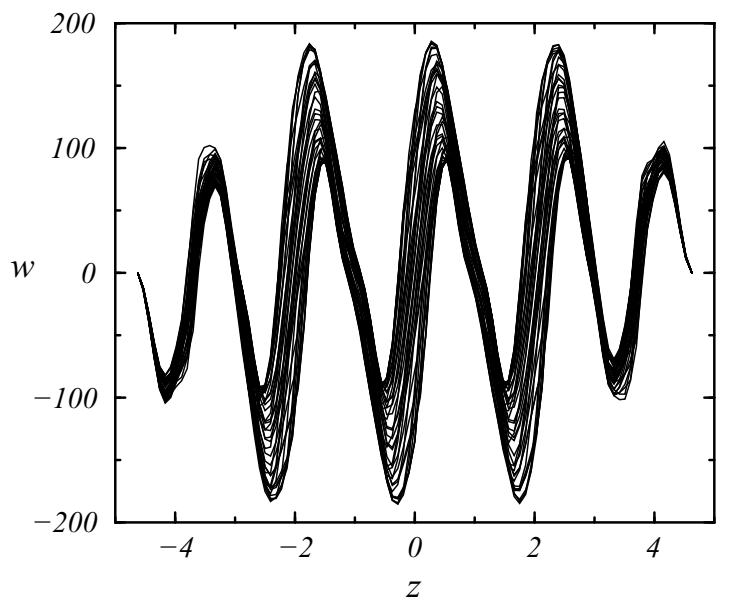

FIG. 4. (Color online) Large jet mode at $\operatorname{Re}=720$ and $\Gamma=9.25$. Contours of angular momentum $r v$, (a) on a cylindrical surface $(\theta, z) \in$ $[0,2 \pi] \times[-\Gamma / 2, \Gamma / 2]$ at $r=1.5$ and (b) on a meridional plane $(r, z) \in[1,2] \times[-\Gamma / 2, \Gamma / 2]$ at $\theta=\pi$. (c) Overlapped axial velocity profiles $w$ over the line $r=1.8, \theta=\pi, z \in[-\Gamma / 2, \Gamma / 2]$, corresponding to 40 different time steps.

of the outgoing jets. The frequency of the LJ remains around $56 \%$ of the rotation frequency of the inner cylinder. All these observations coincide with previously reported descriptions of this flow pattern $[14,42,43]$. The LJ preserves the same discrete symmetry group $Z_{2}$ as the $\mathrm{SJ}$.

\section{Very low frequency mode}

With the increase of Re there is a small phase shift in the oscillations of contiguous vortices. This phase difference results in small wavelength disturbances which slowly propagate in the axial direction, giving rise to the appearance of an axisymmetric mode $m=0$, known as a very low frequency mode (VLF). The resulting quasiperiodic state was first reported by Gerdts et al. [14], and characterized in detail by Von Stamm et al. [15] in a subsequent experimental study. The discrete central reflection retained by the $\mathrm{SJ}$ and $\mathrm{LJ}$ is broken due to the occurrence of VLF. This is illustrated in Fig. 5 at $\operatorname{Re}=780$ and $\Gamma=9$. It shows contours of angular momentum on two opposite (differing by an angle of $\pi$ ) meridional planes, the second plane (b) being vertically reflected $(z \rightarrow-z)$. If the central inversion symmetry were preserved, both figures should be identical, however, there are small differences, mainly around the second and fourth outgoing jets.
The dynamical behavior of the VLF is very sensitive to changes in $N$ and $\Gamma$. Abshagen and Pfister [44] experimentally found a transition between two types of VLF with different features at $\Gamma / N=0.907$. Below this value, the onset of the VLF occurs via a homoclinic bifurcation, whereas for $\Gamma / N>$ 0.907 it takes place for larger values of Re through a Hopf bifurcation, resulting in an oscillation frequency one order of magnitude larger than that in the homoclinic case. The scenarios of transition to chaos also vary among the different experimental studies. For example, in [15], the flow undergoes a period-doubling route to chaos, whereas in [44], a transition to chaos via spatiotemporal intermittency was found.

In the study here undertaken, the VLF emerges from a Hopf bifurcation and the flow approaches chaos via the perioddoubling bifurcation cascade which is sketched in Fig. 6. The sequence of states that occur throughout the period-doubling route to chaos cannot be identified by visual inspection of the flow patterns, but requires the analysis of the time series recorded over a long simulation time. The different states of this sequence are shown in Fig. 7. Figure 7(a) illustrates the VLF state near the bifurcation $(\operatorname{Re}=770)$. The frequency spectrum (first column) shows the large difference between the frequencies of the underlying rotating wave, in this case the $\mathrm{SJ}$, and the frequency of VLF, $f_{p}$ and $f_{\mathrm{VLF}}$, respectively. An increase in the power spectral density (PSD) of the harmonics of $f_{\mathrm{VLF}}$ near $f_{p}$ is another distinctive mark of these solutions.

(a)

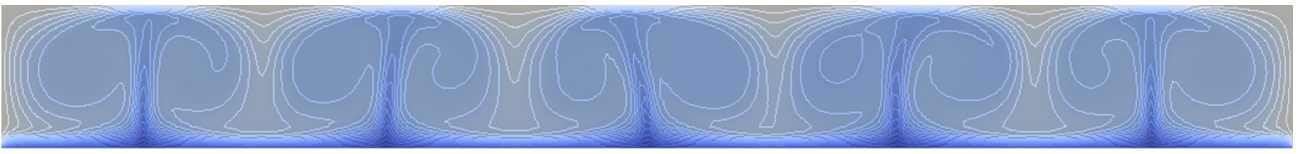

(b)

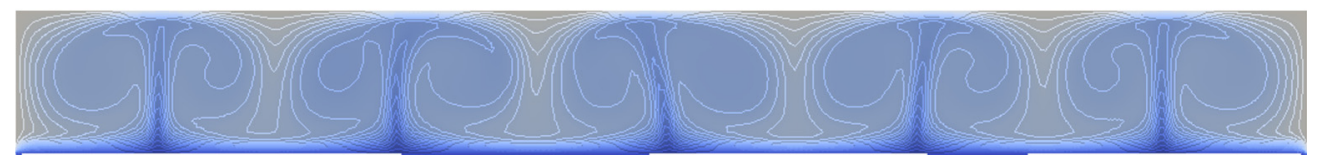

FIG. 5. (Color online) VLF state at $\operatorname{Re}=780$ and $\Gamma=9$. (a) Contours of angular momentum on a meridional plane $(r, z) \in[1,2] \times$ $[-\Gamma / 2, \Gamma / 2]$ and $\theta=\pi$. (b) The inverse symmetric of (a) $(\theta \rightarrow \theta+\pi$ and $z \rightarrow-z)$. There are 20 contours in $[0,780]$. 


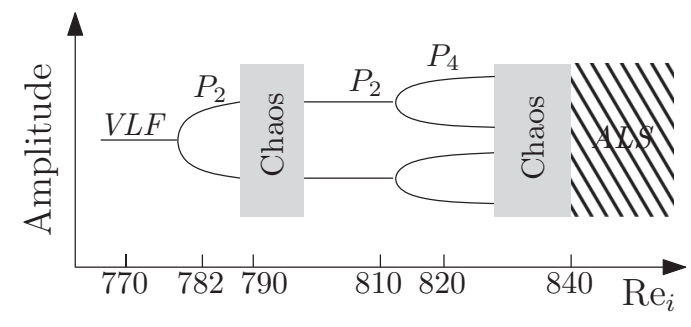

FIG. 6. Schematic of the period-doubling route to chaos exhibited by the VLF as Re is increased, for $\Gamma=9$. The solutions at the $\operatorname{Re}$ indicated are described in Fig. 7.

The phase portrait of the trajectories defined by the axial velocities $w_{1}$ and $w_{2}$ in the phase plane is displayed in the second column. It reflects the toroidal structure of the VLF state due to the actions of $f_{p}$ and $f_{\mathrm{VLF}}$. To facilitate the visual interpretation of the phase portraits, we have also computed the intersections of these trajectories with a carefully chosen plane, the so-called Poincare section, in which the action of $f_{p}$ is removed. This is depicted in the third column, showing a single closed orbit corresponding to the low frequency motion. The VLF doubles its period ( $P$ to $P 2$ ) for the first time at $\operatorname{Re} \approx 782$. The frequency spectrum in Fig. 7(b) illustrates the emergence of the new frequency $f_{\mathrm{VLF}_{2}}=f_{\mathrm{VLF}} / 2$. Since the dynamics is entirely dominated by the very low frequency mode, only the low frequency spectrum is shown in this and the subsequent states of the sequence. The period-doubling bifurcation can also be easily observed in the Poincare section, where a new periodic orbit emerges, with twice the period of the original orbit. Further increasing Re, the flow becomes weakly chaotic at $\mathrm{Re} \approx 788$, which is illustrated in Fig. 7(c), and remains disordered up to $\mathrm{Re} \approx 810$. Here, the flow recovers the period $P 2$ [see Fig. 7(d)], however, a comparison between the phase portraits in Figs. 7(b) and 7(d) reveals that the structure of the new state differs from that before the chaotic regime. A subsequent increase in Re leads to a new period doubling $(P 4)$, $f_{\mathrm{VLF}_{4}}=f_{\mathrm{VLF}_{2}} / 2$, which is illustrated in Fig. 7(e) at $\mathrm{Re}=820$. The phase portrait resembles that in Fig. 7(d), nevertheless, the enlargement of the phase trajectories is reflected in the fact that, for the same simulation time in both cases, the torus surface in Fig. 7(e) has not been filled yet. The flow becomes chaotic again with further increase in Re. This flow regime is shown at $\operatorname{Re}=840$ in Fig. 7(f). Finally, the chaotic behavior disappears at $\mathrm{Re} \approx 855$ and gives rise to the regime of axially localized states which is discussed in the next section.

\section{AXIALLY LOCALIZED STATES}

\section{A. Description}

A new pre-turbulent flow regime arises for $\mathrm{Re} \geqslant 855$ and $8.6<\Gamma<9.5$. The resulting flow patterns are characterized by the existence of large amplitude oscillations localized on some, but not all, of the outgoing jets of the bulk vortices. Due to this particularity we refer to these flow structures as axially localized states (ALS). The main consequence of this localization is the appearance of multiple steady states, differing in the axial position of the outgoing jet (or jets) showing the large amplitude oscillations. Thus, a remarkably high number of ALS can be observed as increasing the number of vortices in the flow. The ALS were found experimentally by Abshagen et al. [12], who reported the existence of a wide spectrum of ALS for several $N$-vortex flows, many of which coexist in the parameter space, giving rise to a very complex and intriguing dynamics. In this section, apart from confirming numerically these experimental results, we focus on the transitions taking place within this region of the parameter space, which eventually lead to chaotic behavior.

The family of ALS numerically obtained for the $N=10$ vortex flow is illustrated in Fig. 8 through a three-dimensional contour of angular momentum $r v=-450$, which reflects the axial localization of the large jet oscillations, and a plot such as that in Figs. 3(c) and 4(c), which gives a clear idea of the difference in amplitude between the localized large jet oscillations and the oscillations in the rest of the vortices. As occurred in [12], not all possible ALS (six in the case of a 10-vortex flow) have been found. This could mean that the remaining ALS are unstable, or simply that very specific sequences of states, which have not been detected in this study, are required to reach these states. To denote the different ALS, we have adopted the nomenclature used in [12]. Each ALS can be expressed with a binary number where the outgoing jets with large amplitude oscillations are indicated with ones, and the rest of vortices are denoted by zeros. This binary number is converted into a decimal base, starting from the Ekman vortex at the top, and placed as a subscript of ALS. For example, the binary number corresponding to the state in Fig. 8(c) is 01000 because the only pair of vortices oscillating with large amplitude is the second one starting from the bottom. It is subsequently converted to decimal base (8) and the state is referred to as $\mathrm{ALS}_{8}$. Depending on the location of the large jet oscillations, the ALS may or not preserve the axial reflection symmetry.

The symmetric states in Figs. 8(a) and 8(b), denoted by $\mathrm{ALS}_{10}$ and $\mathrm{ALS}_{4}$, respectively, can be obtained as a direct transition from any of the states described in Sec. III. In contrast, the asymmetric states $\mathrm{ALS}_{8}$ and $\mathrm{ALS}_{2}$, shown in Figs. 8(c) and 8(d), are only found when starting the simulation from other ALS. Figure 9(a) shows the transition curves observed in experiments [12] for $N=8$ (thick lines) and $N=12$ (thin lines). The transition from TVF to small jet states is the cusp (dot-dashed lines) at the lower-right corner. The transition from SJ to LJ (dashed lines) takes place for moderate Reynolds number $(\operatorname{Re} \lesssim 900)$, while for larger values $(\operatorname{Re} \gtrsim 900)$ the transition (long-dashed lines) is from SJ to localized ALS. The solid curves are the lower limit of the region of existence of the ALS. This region is hysteretical, so different ALS, and also nonlocalized states (SJ, LJ, VLF), coexist within it. The collapse of data corresponding to $N=8$ and $N=12$ number of vortices is remarkable, and it is more pronounced for larger values of $N$. We have added to this Fig. 9(a) three one-dimensional paths, shown as straight lines with an arrow. Although we have performed a comprehensive exploration of the parameter region shown in this figure, we present in the paper detailed results of the different transitions that take place, only along these three paths. The transitions corresponding to the path for fixed $\Gamma=9$ and moderate Reynolds numbers $(\mathrm{Re} \sim 800)$ do not involve ALS and have already been discussed in Sec. III C (Figs. 6 and 7). The other 

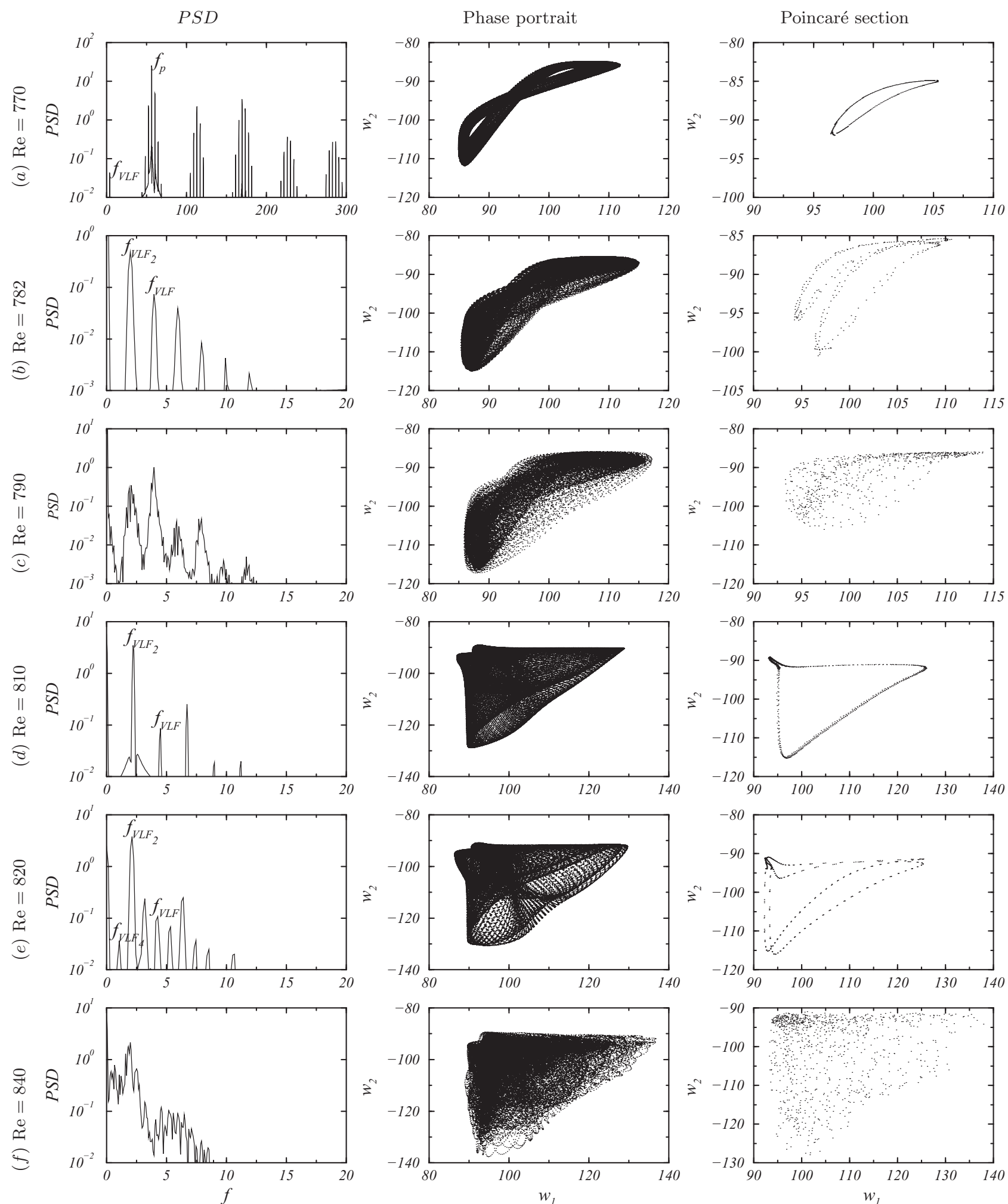

FIG. 7. Power spectral density PSD, phase portrait, and Poincare section illustrating the sequence of period-doubling bifurcations sketched in Fig. 6 at $\Gamma=9$. (a) VLF at $\operatorname{Re}=770$, near the bifurcation; (b) first period doubling $P 2$ of $\mathrm{VLF}$ at $\operatorname{Re}=782$; (c) first chaotic solution at $\operatorname{Re}=790$; (d) state with period $P 2$ but different structure at $\operatorname{Re}=810$; (e) second period doubling $P 4$ of VLF at $\operatorname{Re}=820$; (f) chaotic flow at $\mathrm{Re}=840$.

two paths correspond to transitions involving ALS, and are analyzed in Sec. IV B.

Figure 9(b) shows the states achieved in the simulations performed for $\operatorname{Re}>850$, overlapped in the parameter space with the experimentally measured critical boundaries for $N=8$ in Fig. 9(a). It can be seen that all numerical results lie in parameter space consistently with experiments. There is a substantial overlap among the different ALS, so that 
(a)

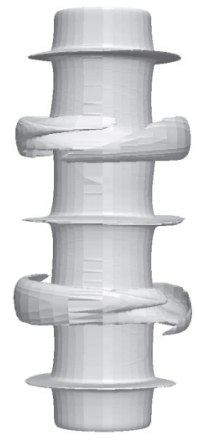

(c)

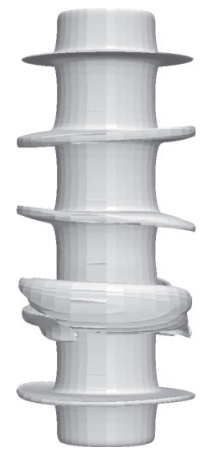

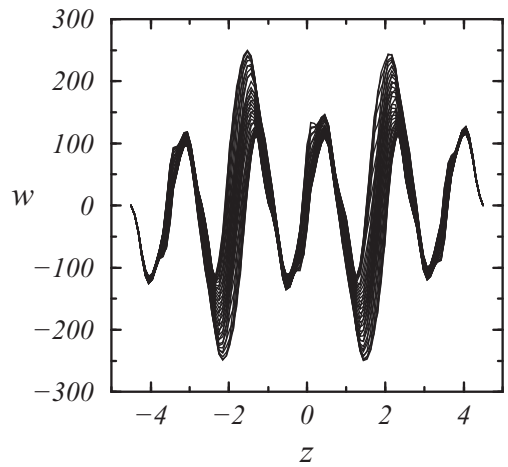

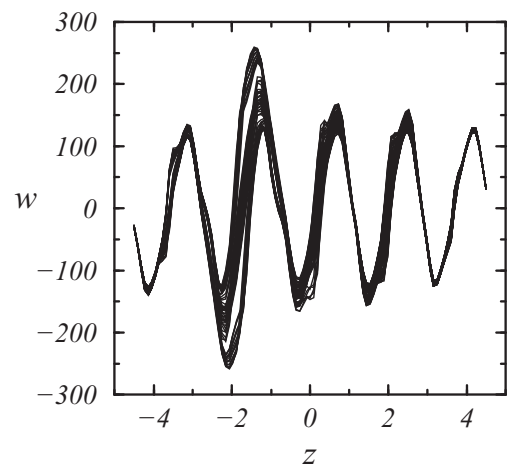

(b)
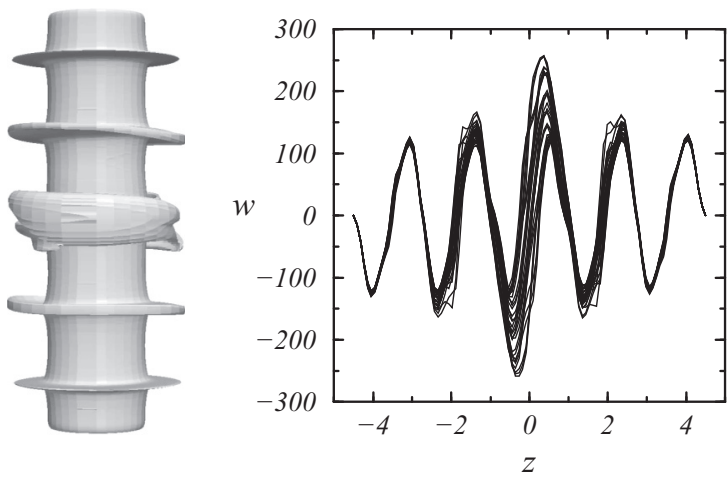

(d)

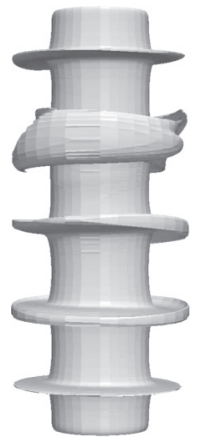

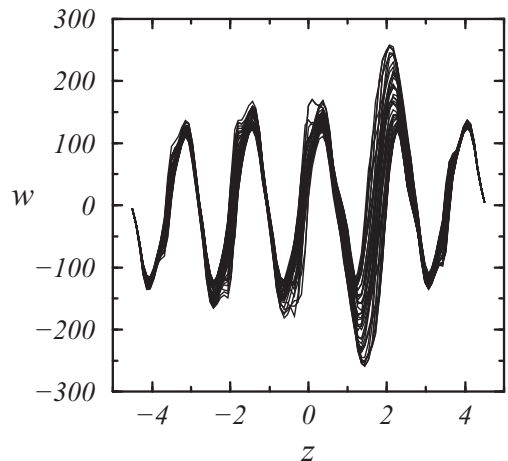

FIG. 8. Three-dimensional contour of the angular momentum $(r v=-450)$ and axial velocity profiles $w$ recorded at $r=1.8, \theta=\pi$, and $z \in[-\Gamma / 2, \Gamma / 2]$ for 40 different time steps and overlapped on the same plot. (a) $\mathrm{ALS}_{10}$ at $\mathrm{Re}=920$ and $\Gamma=9$, (b) $\mathrm{ALS}_{4}$ at $\operatorname{Re}=960$ and $\Gamma=9$, (c) $\mathrm{ALS}_{8}$ at $\mathrm{Re}=960$ and $\Gamma=9.1$, and (d) $\mathrm{ALS}_{2}$ at $\mathrm{Re}=960$ and $\Gamma=9.3$. The nomenclature $\mathrm{ALS}_{\text {number }}$ is explained in the text.

obtaining each of them critically depends on the path followed in parameter space. The $\mathrm{ALS}_{10}$, indicated with diamonds in Fig. 9, is the only one of the computed ALS where two outgoing jets oscillate with large amplitude. It is the most stable ALS for the lowest values of $\operatorname{Re}(855 \leqslant \operatorname{Re} \leqslant 1050)$ in which the regime of ALS takes place. This is in agreement with [12], where the states with the highest number of axially localized large jet oscillations were also found at the lowest values of Re. Also in accordance with these experimental results is the fact that all ALS with large jet oscillations localized on a (a)

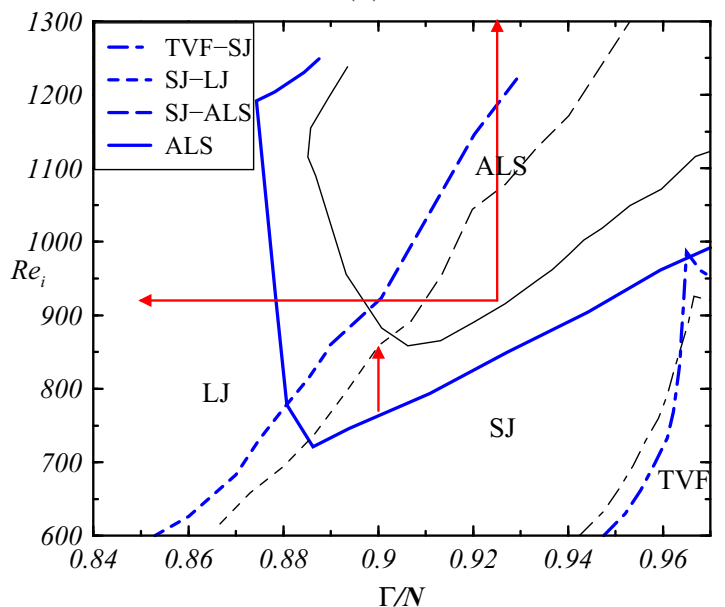

(b)

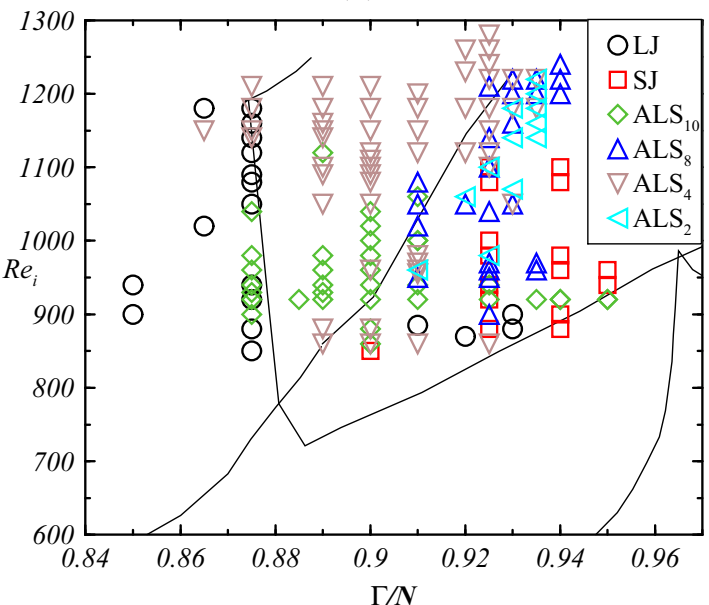

FIG. 9. (Color online) (a) Experimental transition curves between TVF, SJ, LJ, and ALS as indicated, for $N=8$ (thick lines, blue online) and $N=12$ (thin lines, black online); data from [12, Figs. 5 and 7]. Straight lines with arrow (red online) correspond to the transitions shown in Figs. 6 and 11. (b) Distribution in the parameter space of the numerically computed flow patterns for Re $>850$. The solid lines represent the experimental stability boundaries for the case of $N=8$ shown in (a). 
(a)

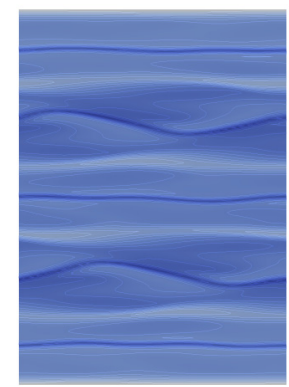

(c)

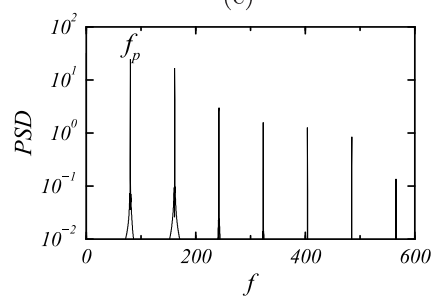

(b)

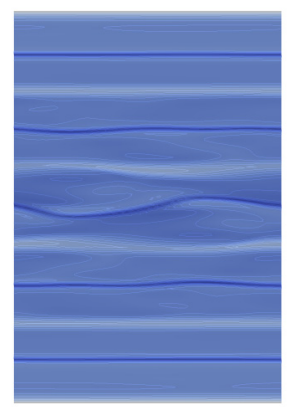

(d)

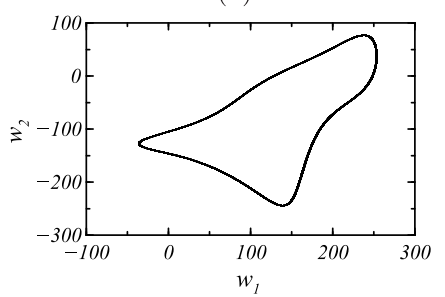

FIG. 10. (Color online) Contours of angular momentum $r v$ on a cylindrical surface $(\theta, z) \in[0,2 \pi] \times[-\Gamma / 2, \Gamma / 2]$ at $r=1.5$ for (a) $\mathrm{ALS}_{10}$ at $\mathrm{Re}=920$ and $\Gamma=9.25$; and (b) $\mathrm{ALS}_{4}$ at $\mathrm{Re}=1260$ and $\Gamma=9.25$; (c) and (d) are, respectively, the power spectral density (PSD) and phase portrait for $\mathrm{ALS}_{10}$ at $\mathrm{Re}=920$ and $\Gamma=9.25$.

single outgoing jet have been found. The symmetric $\mathrm{ALS}_{4}$, marked with down-triangles in Fig. 9, is very stable in the range $1050 \leqslant R e \leqslant 1280$, whereas the states $\mathrm{ALS}_{8}$ and $\mathrm{ALS}_{2}$, indicated with left and up-triangles, respectively, are found when considering longer systems $(9.1 \leqslant \Gamma \leqslant 9.4)$. All ALS can be found hysteretically, coexisting with the SJ and LJ in some regions of the parameter space, as is shown in Fig. 9(b).

The formation of a certain ALS obeys to a specific stable arrangement of the wavelengths of the vortex pairs within a system of finite length. However, the phases of oscillation of the different ALS exhibit the same coherence as for the LJ state. This can be seen from Figs. 10(a) and 10(b), which show the contours of angular momentum on a cylindrical surface for the $\mathrm{ALS}_{10}$ and $\mathrm{ALS}_{4}$. In both figures the outgoing jets oscillate in phase (although it is quite difficult to visually distinguish this feature between contiguous jets with large and small amplitude oscillation) and weak oscillating ingoing jets are in antiphase with the oscillations of the outgoing jets. While the ALS preserve this phase coherence they are found as rotating waves in parameter space, precessing with the same oscillation frequency, $f_{p} \approx 55 \%$ of the rotation frequency of the inner cylinder, as the LJ state. Figures 10(c) and 10(d) show the frequency spectrum and phase portrait of the $\mathrm{ALS}_{10}$ at $\operatorname{Re}=920$ and $\Gamma=8.85$, clearly illustrating its behavior as rotating wave. As in Sec. III C, the breaking of the phase coherence of the different vortex pairs leads to the emergence of the VLF, which coexists with the ALS in a wide range of control parameters, and plays a crucial role in the transitions to chaos described below.

\section{B. Transition to chaos}

As a consequence of the diversity of states coexisting in the parameter space there exist multiple routes to chaos. Each one is determined by the initial state and the path followed in parameter space, differing from the others in the number of transitions and types of ALS arising. Nevertheless, despite the complexity introduced by the coexistence of states, the dynamical behavior associated with the transitions is similar in all cases and depends essentially on the control parameter that is varied. Figure 11 is a schematic of the bifurcations found starting from the $\mathrm{ALS}_{10}$ state at $\Gamma=9.25$ and $\mathrm{Re}=920$, when decreasing $\Gamma$ at constant Re [Fig. 11(a)], and when increasing Re at constant $\Gamma$ [Fig. 11(b)]. These two one-dimensional paths in $(\operatorname{Re}, \Gamma)$ parameter space correspond to the straight lines with arrow starting at $\Gamma=9.25$ and $\operatorname{Re}=920$ in Fig. 9(a). The $\mathrm{ALS}_{10}$ initial state of both sequences is a rotating wave with azimuthal drift frequency $f_{p}$ shown in Figs. 10(c) and 10(d).

Figure 12 shows the different states found in the bifurcation scenario occurring when $\Gamma$ is reduced [Fig. 11(a)]. The initial state $\mathrm{ALS}_{10}$ is stable up to $\Gamma=8.84$, where the transition illustrated in Fig. 12(a) takes place. The frequency spectrum (PSD) suggests that the coupling between the oscillations of the different vortex pairs is broken simultaneously with the period doubling of the main frequency $f_{p}$, resulting in the appearance of two frequencies $f_{p_{21}}$ and $f_{p_{22}}$, with similar amplitudes and equidistant from the value $f_{p_{2}}=f_{p} / 2$, corresponding to the frequency of the period doubling. This period-doubling bifurcation with splitting of the corresponding frequency is indicated by $P_{2}^{*}$ in Fig. 11. The small difference between $f_{p_{21}}$ and $f_{p_{22}}$ results in a very low frequency $f_{\mathrm{VLF}}$ which involves, similarly to the VLF described in Sec. III, the axial propagation of the wavelength disturbances of the different vortex pairs. Hence, $f_{p_{21}}$ and $f_{p_{22}}$ can be expressed in terms of $f_{\mathrm{VLF}}$ and $f_{p}$, as $f_{p_{22}}=\left(f_{p}-f_{\mathrm{VLF}}\right) / 2$ and $f_{p_{21}}=$ $\left(f_{p}+f_{\mathrm{VLF}}\right) / 2$. It should be noted that $f_{\mathrm{VLF}}$ is in this case nearly twice $f_{\mathrm{VLF}}$ in the VLF state. The fact that there are (a)

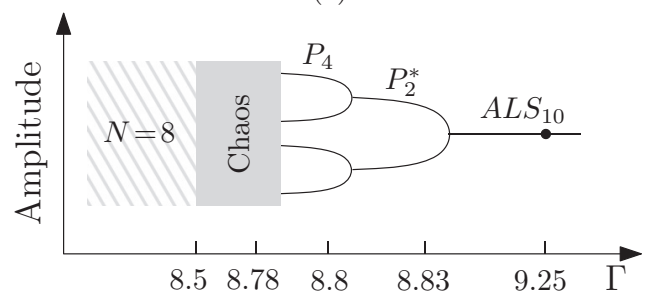

(b)

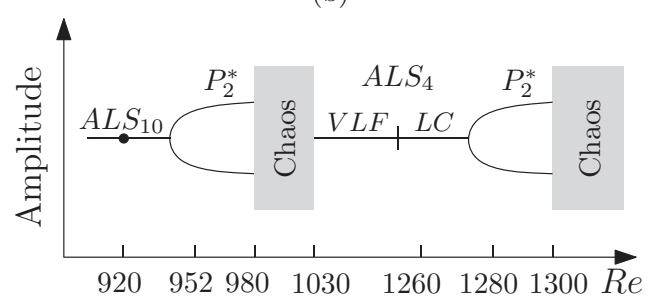

FIG. 11. Schematic of the transitions to chaos exhibited by the $\operatorname{ALS}_{10}$ (a) as $\Gamma$ is decreased, at $\operatorname{Re}=920$, and (b) as Re is increased, at $\Gamma=9.25$. The solutions (a) at the $\Gamma$ indicated are described in Fig. 12, and those in (b) at the Re indicated are described in Fig. 13. The initial state, indicated as a solid disk, is the $\mathrm{ALS}_{10}$ state at $\Gamma=9.25$ and $\mathrm{Re}=920$. 

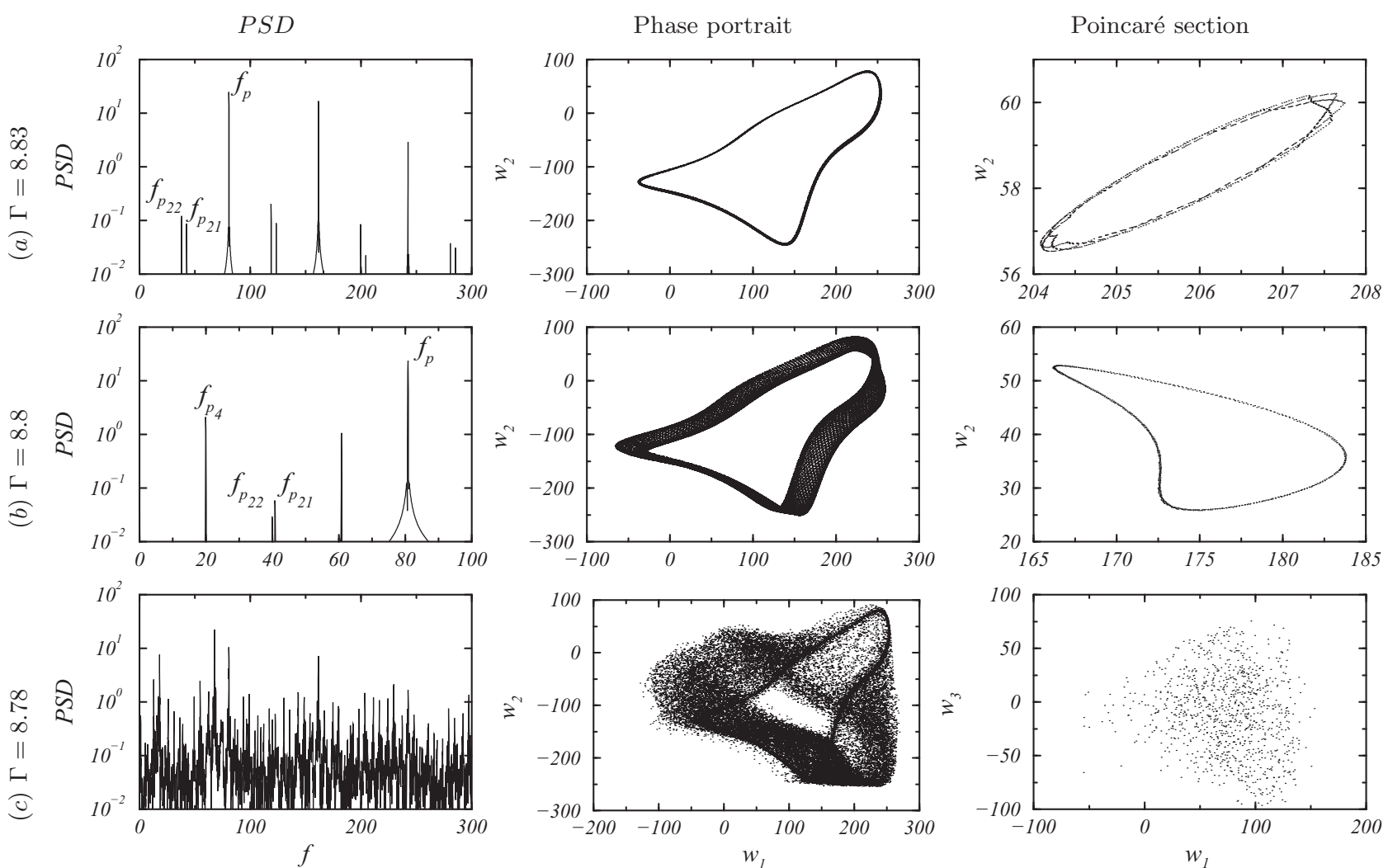

FIG. 12. Power spectral density, phase portrait, and Poincaré section illustrating the sequence of states in the route to chaos when Re $=920$ and $\Gamma$ is decreased. The initial state is the $\mathrm{ALS}_{10}$ at $\operatorname{Re}=920$. (a) State resulting from the first transition at $\Gamma=8.83$; (b) state resulting from the second transition at $\Gamma=8.80$; (c) weakly chaotic flow at $\Gamma=8.78$.

two frequencies of similar amplitude arising at the same time is probably related to the existence of two vortex pairs with large jet oscillations in the $\mathrm{ALS}_{10}$. The new state is therefore a two-dimensional torus in the phase space. Nevertheless, the amplitude of the main frequency is much higher than that for the additional frequency, so that the phase portrait resembles a limit cycle. The intersection of the invariant torus with a Poincare section shows a closed orbit with three loops. This reflects the coupling between the mechanism responsible for the period doubling of the main frequency and the oscillation due to the very low frequency mode. Due to the small region of the phase plane in which the orbit is located, it is quite difficult to distinguish the full trajectory of the orbit in Fig. 12(a). As far as we know this transition has not been previously reported, however, there exist a few works in the realm of the dynamical systems theory that analyze a problem resembling the transition described above. They discuss the influence of a periodic perturbation in a nonlinear system which is close to a period-doubling bifurcation [45-47]. In all of these studies, the dynamics is entirely dominated by the periodic perturbation, which suppresses the period-doubling bifurcation. This behavior is somewhat similar to that of the aforementioned bifurcation, with $f_{\mathrm{VLF}}$ playing the dynamical role of the periodic perturbation.

When $\Gamma$ is reduced from 8.84 to approximately 8.81 , the amplitudes of $f_{p_{21}}, f_{p_{22}}$, and $f_{\mathrm{VLF}}$ gradually increase. For $\Gamma$ values below 8.81, $f_{p}$ undergoes a second period-doubling bifurcation, which is clearly illustrated in Fig. 12(b). In this case, unlike the first period doubling, there is a single frequency arising at $f_{p_{4}}=f_{p} / 4$, whose amplitude is substantially larger than that for $f_{p_{21}}$ and $f_{p_{22}}$. This transition seems to help restore the phase coherence of the initial state. This is reflected in the fact that $f_{p_{21}}$ and $f_{p_{22}}$ closely approach $f_{p_{2}}$, so $f_{\mathrm{VLF}}$ becomes approximately zero. Nevertheless, the flow is still quasiperiodic, as indicates the limit cycle displayed in the Poincare section. The phase portrait reveals the same initial spatial structure, which becomes increasingly thicker as the flow approaches chaos. When we further reduce $\Gamma$, the flow becomes suddenly chaotic at $\Gamma=8.78$. This behavior is easy to identify in Fig. 12(c). For example, the Poincare section clearly shows the loss of periodicity of the previous states, giving rise to a cloud of points in the phase space. The flow remains chaotic up to $\Gamma \approx 8.5$, where it bifurcates to a more stable state with $N=8$ vortices. It is noticeable that all transitions happen in a very short range $8.78 \leqslant \Gamma \leqslant 8.84$. This sequence of transitions to chaos as $\Gamma$ is decreased has only been found for $\mathrm{Re} \leqslant 950$, when using $\mathrm{ALS}_{10}$ as the initial state. For higher $\mathrm{Re}$ or a different initial state, the flow approaches chaos similarly when reducing Gamma or increasing Re, as is described below.

Figure 13 shows the sequence of bifurcations to chaos that occurs when Re is increased and $\Gamma=9.25$ [Fig. 11(b)]. The initial state is the same as in the transition described above, the $\mathrm{ALS}_{10}$ at $\mathrm{Re}=920$. This state loses stability at $\mathrm{Re}=948$, resulting in a similar state to that obtained after the first transition when decreasing $\Gamma$. The frequency spectrum in Fig. 13(a) (corresponding to $\operatorname{Re}=952$ ), displays the same 

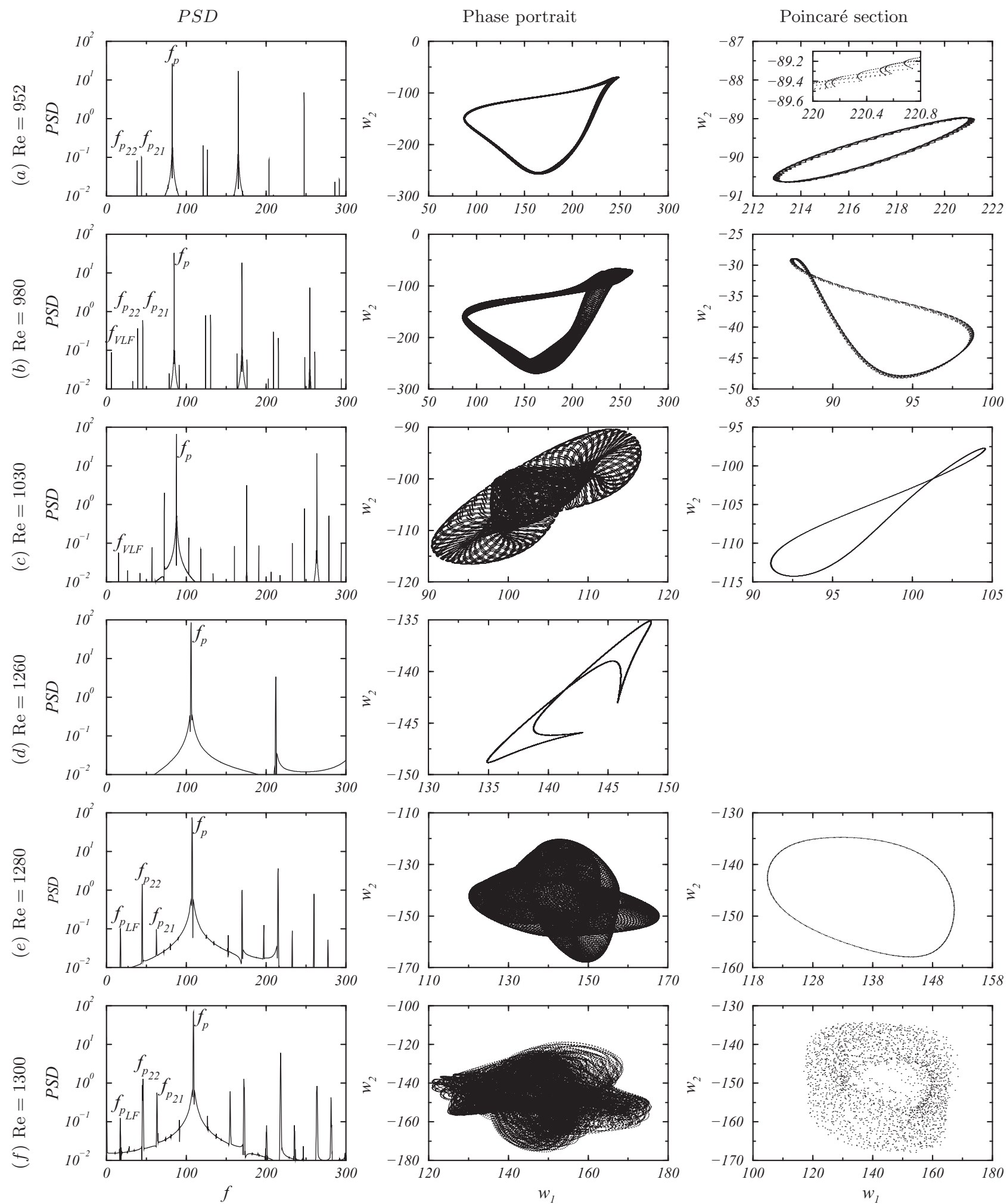

FIG. 13. Power spectral density, phase portrait, and Poincaré section illustrating the route to chaos when Re is increased and $\Gamma=9.25$ is fixed. The initial state is the $\mathrm{ALS}_{10}$ at $\mathrm{Re}=920$. (a) State resulting from the first transition at $\operatorname{Re}=952$; (b) intermediate state before the transition to the $\mathrm{ALS}_{4}$ at $\mathrm{Re}=980$; (c) $\mathrm{ALS}_{4}$ with a low frequency mode at $\mathrm{Re}=1030$; (d) $\mathrm{ALS}_{4}$ as rotating wave at $\mathrm{Re}=1260$. (e) Emergence of the low frequency mode at $\operatorname{Re}=1280$. (f) Chaotic flow at $\operatorname{Re}=1300$.

behavior as in Fig. 12(a). Again, it is observed that two additional frequencies, $f_{p_{21}}$ and $f_{p_{22}}$, arise in the vicinity of the frequency $f_{p_{2}}=f_{p} / 2$, corresponding to the period doubling of the main frequency $f_{p}$. These frequencies cause the appearance of a very low frequency, $f_{\mathrm{VLF}}=f_{p_{21}}-f_{p_{22}}$, which is about twice $f_{\mathrm{VLF}}$ of the VLF state found in Sec. III. The phase portrait and Poincaré section in Fig. 13(a) are also qualitatively similar to those in Figs. 12(b) and 12(c). 
A closeup has been included in the Poincaré section in order to discern the complex path followed by the periodic orbit. The amplitude of $f_{\mathrm{VLF}}$ grows progressively with the increase of Re. This fact, which is reflected in Fig. 13(b) at $\mathrm{Re}=980$, might be ascribed to the increasing phase difference between the oscillations of contiguous vortices with respect to the initial state, which result in larger wavelength disturbances. Further increasing Re the $\mathrm{ALS}_{10}$ becomes weakly chaotic, and subsequently bifurcates into another axially localized state which is more stable at higher values of Re, the $\mathrm{ALS}_{4}$. This transition, which takes place at $\operatorname{Re}=1030$, is illustrated in Fig. 13(c). The existence of a new state can be seen from the phase portrait, which reveals an entirely different structure with respect to that in Figs. 13(a) and 13(b). There also exist a low frequency $f_{\mathrm{LF}}$ coexisting with the frequency of the rotating wave, which is one order of magnitude higher than $f_{\mathrm{VLF}}$ in the previous state. The dynamics is still on a $2 \mathrm{~d}$ torus surface, as is reflected by the single closed orbit shown in the Poincare section. The amplitude of $f_{\mathrm{LF}}$ decreases with the increase of $\mathrm{Re}$, so the $\mathrm{ALS}_{4}$ progressively turns into a rotating wave. This happens at $\mathrm{Re} \approx 1130$, where $f_{\mathrm{LF}}$ completely disappears and the ensuing flow pattern is a pure rotating wave with frequency $f_{p}$. This behavior, which is illustrated in Fig. 13(d) at $\operatorname{Re}=1260$, persists up to $\operatorname{Re} \approx 1275$. Note that the Poincaré section gives in this case a single point in phase space, and thus it has not been plotted. When $\operatorname{Re}>1275$ the coupling between neighboring vortices is again broken, resulting in a similar mechanism to that described for the first transition. Figure 13(e) illustrates this state at $\mathrm{Re}=1280$. It is observed that, like the first transition, there arise two peaks at $f_{p_{21}}$ and $f_{p_{22}}$, which are equidistant to $f_{p} / 2$, and their difference leads to the appearance of a low frequency $f_{\mathrm{LF}}$. Since the difference between $f_{p_{21}}$ and $f_{p_{22}}$ is significantly larger than in the first transition, $f_{\mathrm{LF}}$ is approximately one order of magnitude higher than $f_{\mathrm{VLF}}$. Moreover, the amplitude of $f_{p_{22}}$ near the bifurcation is much larger than that of $f_{p_{21}}$, in contrast to the first transition, where both frequencies emerged approximately with the same amplitude. This could be related to the existence of a single outgoing jet oscillating with large amplitude in the $\mathrm{ALS}_{4}$. Note that, unlike Figs. 12(a) and 13(a), the Poincaré section does not reflect a doubled loop, which is probably due to dominance of $f_{p_{22}}$ over $f_{p_{21}}$. Subsequently increasing Re, $f_{\mathrm{LF}}$, and $f_{p_{21}}$ increase progressively their amplitude and additional frequencies come into play, causing eventually the appearance of chaos. The flow becomes first weakly chaotic at $\operatorname{Re}=1300$, as is shown in Fig. 13(f).

\section{CONCLUSION}

The bifurcation scenario in a wide gap $\left(r_{i} / r_{o}=0.5\right)$ TaylorCouette apparatus, where only the inner cylinder is rotating and the length-to-gap aspect ratio is restricted to the range $8.5<\Gamma<9.5$, has been numerically investigated. We focus on the dynamics of recently reported experimental flow patterns [12] which exhibit large amplitude oscillations localized in some of the outgoing jets of the bulk vortices. We refer to these states as axially localized states ALS.

We find that, for the particular case of a 10-vortex flow, four different ALS coexist in parameter space, differing in the axial position where the large amplitude oscillations take place. We have not found all possible ALS for this flow (6), but consistently with the experiments, all ALS having a single outgoing jet oscillating with large amplitude occur. There is only one ALS with large amplitude oscillations in two outgoing jets, which is the most stable ALS at the lowest values of Re in the region of the parameter space where the ALS are located. The states that retains the axial reflection symmetry are more stable than the asymmetric states, and thus are found in larger areas of the parameter space. These results are also in full agreement with experimental observations [12].

Each ALS is related to a specific stable coupling of the vortex pairs in a finite-length system. Varying the control parameters ( $\Gamma$ and $\mathrm{Re}$ ) modifies the wavelengths of the different vortex pairs, generally increasing (decreasing) in those vortex pairs where the large amplitude oscillations manifest, and in return, decreasing (increasing) in the Ekman vortices. While the coupling between the vortices remains, the ALS are rotating waves with a single frequency that coincide with that for the so-called large jet state LJ [14], in which all outgoing jets in the bulk flow oscillate with large amplitude. Nevertheless, when the coupling is broken there arise disturbances in the wavelength of the different vortex pairs that slowly spread in the axial direction, similarly to the situation described in [14], who termed the resulting axisymmetric motion as very low frequency mode VLF. The growth of these disturbances determines the flow transitions occurring as the control parameters are varied, leading to either other ALS or the appearance of chaos.

We observed that, in all cases, the VLF seems to emerge almost simultaneously with the period doubling of the orbit associated with the frequency of the $\operatorname{ALS} f_{p}$, resulting in the splitting of $f_{p} / 2$ in two very close frequencies, $f_{p_{21}}$ and $f_{p_{22}}$, separated $\pm f_{\mathrm{VLF}} / 2$ from it. After this transition, the behavior has been found to depend on the control parameter that is changed. Decreasing $\Gamma$ (when $\operatorname{Re}<950$ ) results in restoring the phase coherence between vortex pairs, so $f_{\mathrm{VLF}}$ approaches zero and the system undergoes a second period-doubling bifurcation, which precedes the final transition to chaos. In contrast, when Re is increased, the value of $f_{\mathrm{VLF}}$ as well as its amplitude gradually increase, leading to a distinct ALS or, at a sufficiently high Re, to the occurrence of chaos. This behavior is also observed as reducing $\Gamma$ for $\operatorname{Re}>950$. The behavior of the VLF mode is very sensitive to changes in the control parameters. It has been found to occur through distinct types of bifurcations, and the amplitude of $f_{\mathrm{VLF}}$ substantially increases with $\operatorname{Re}[15,44]$. There also exist experimental evidences suggesting different dynamical behaviors of the VLF when the number of vortices in the flow is greater than 20 [15]. Consequently, it is likely that different transitions from those described in this paper will arise in systems with higher $\Gamma$.

The excellent agreement between calculations and experimental results strongly suggests that the VLF and ALS states are not due to imperfections and/or noise, that are absent in our simulations, but they are intrinsic features of the Taylor-Couette system. The exact details of the dynamics described in this paper are not easily discernible in laboratory experiments. A large number of the bifurcations presented take place with variations below $1 \%$ in $\operatorname{Re}$ or $\Gamma$, and they involve long transients. Hence, experimental observation of such transitions would require a very precise control of 
parameters over extended periods of time. There is thus a possibility that some of these transitions are sensitive to imperfections. Additional experiments and numerical simulations including weak symmetry breaking are necessary to settle this question.

The dynamics observed are a result of the interaction between flow and end walls and significantly differ from those in simulations using axially periodic boundary conditions. An example of the strong end effects in Taylor-Couette systems is found by comparing the transition between Taylor and spiral vortices when axially periodic [48] and physical no-slip boundary conditions [49] are used. It is expected that end effects will decrease with the increase of $\Gamma$, although they will influence the dynamics even in systems with very large $\Gamma$ $[34,50]$. It would be interesting to determine the value of $\Gamma$ for which some of these effects are negligible and the dynamics resemble those of an axially periodic system. Nevertheless, the computational cost of properly solving extended geometries at high values of Re with our methods is extremely large, if not impossible in many cases. The implementation of multidomain methods, such as that in [51], could help cope with this problem.

\section{ACKNOWLEDGMENTS}

This work was supported by the Spanish Ministerio de Economia y Competitividad, Grants No. FIS2013-40880-P and No. BES-2010-041542. The authors would like to thank Prof. Avila for stimulating discussions. Part of this work was done during a visit of J.M.L. to the Institute of Science and Research (IST) in Klosterneuburg (Austria) whose kind hospitality is warmly appreciated.
[1] J. Dawes, The emergence of a coherent structure for coherent structures: Localized states in nonlinear systems, Proc. R. Soc. London A 368, 3519 (2010).

[2] F. Mellibovsky, A. Meseguer, T. M. Schneider, and B. Eckhardt, Transition in localized pipe flow turbulence, Phys. Rev. Lett. 103, 054502 (2009).

[3] A. P. Willis and R. R. Kerswell, Turbulent dynamics of pipe flow captured in a reduced model: Puff relaminarization and localized edgestates, J. Fluid Mech. 619, 213 (2009).

[4] K. Avila, D. Moxey, A. de Lozar, M. Avila, D. Barkley, and B. Hof, The onset of turbulence in pipe flow, Science 333, 192 (2011).

[5] B. Eckhardt, T. M. Schneider, B. Hof, and J. Westerweel, Turbulence transition in pipe flow, Annu. Rev. Fluid Mech. 39, 447 (2007).

[6] T. M. Schneider, J. F. Gibson, and J. Burke, Snakes and ladders: Localized solutions of plane Couette flow, Phys. Rev. Lett. 104, 104501 (2010).

[7] Y. Duguet, P. Schlatter, and D. S. Henningson, Localized edge states in plane Couette flow, Phys. Fluids B 21, 111701 (2009).

[8] D. Barkley and L. S. Tuckerman, Computational study of turbulent laminar patterns in Couette flow, Phys. Rev. Lett. 94, 014502 (2005).

[9] T. M. Schneider, D. Marinc, and B. Eckhardt, Localized edge states nucleate turbulence in extended plane Couette cells, J. Fluid Mech. 646, 441 (2010).

[10] M. Heise, J. Abshagen, D. Küter, K. Hochstrate, G. Pfister, and C. Hoffmann, Localized spirals in Taylor-Couette flow, Phys. Rev. E 77, 026202 (2008).

[11] J. Abshagen, M. Heise, G. Pfister, and T. Mullin, Multiple localized states in centrifugally stable rotating flow, Phys. Fluids 22, 021702 (2010).

[12] J. Abshagen, J. von Stamm, M. Heise, C. Will, and G. Pfister, Localized modulation of rotating waves in Taylor-Couette flow, Phys. Rev. E 85, 056307 (2012).

[13] C. Jones, The transition to wavy Taylor vortices, J. Fluid Mech. 157, 135 (1985).

[14] U. Gerdts, J. von Stamm, T. Buzug, and G. Pfister, Axisymmetric time-dependent flow in the Taylor-Couette system, Phys. Rev. E 49, 4019 (1994).
[15] J. von Stamm, U. Gerdts, T. Buzug, and G. Pfister, Symmetry breaking and period doubling on a torus in the VLF regime in Taylor-Couette flow, Phys. Rev. E 54, 4938 (1996).

[16] M. Golubitsky and I. Stewart, Symmetry and stabiliy in TaylorCouette flow, SIAM J. Math. Anal. 17, 249 (1986).

[17] K. T. Coughlin and P. S. Marcus, Modulated waves in TaylorCouette flow Part 2. Numerical simulation, J. Fluid Mech. 234, 19 (1992).

[18] M. Gorman and H. L. Swinney, Spatial and temporal characteristics of modulated waves in the circular Couette system, J. Fluid Mech. 117, 123 (1982).

[19] M. Heise, C. Hoffmann, J. Abshagen, A. Pinter, G. Pfister, and M. Lücke, Stabilization of domain walls between traveling waves by nonlinear mode coupling in Taylor-Couette flow, Phys. Rev. Lett. 100, 064501 (2008).

[20] C. Hoffmann, M. Heise, S. Altmeyer, J. Abshagen, A. Pinter, G. Pfister, and M. Lücke, Nonlinear defects separating spiral waves in Taylor-Couette flow, Phys. Rev. E 80, 066308 (2009).

[21] P. Hirschberg and E. Knobloch, Complex dynamics in the Hopf bifurcation with broken translation symmetry, Physica D 90, 56 (1996).

[22] G. Dangelmayr, J. Hettel, and E. Knobloch, Parity-breaking bifurcation in inhomogeneous systems, Nonlinearity 10, 1093 (1997).

[23] M. Westerburg and F. H. Busse, Centrifugally driven convection in the rotating cylindrical annulus with modulated boundaries, Nonlin. Proc. Geophys. 10, 275 (2003).

[24] U. Thiele and E. Knobloch, Driven drops on heterogeneous substrates: Onset of sliding motion, Phys. Rev. Lett. 97, 204501 (2006).

[25] F. Marques, A. Meseguer, J. M. Lopez, J. R. Pacheco, and J. M. Lopez, Bifurcations with imperfect $\mathrm{SO}(2)$ symmetry and pinning of rotating waves, Proc. R. Soc. London, Ser. A 469, 20120348 (2013).

[26] M. Avila, F. Marques, J. M. Lopez, and A. Meseguer, Stability control and catastrophic transition in a forced Taylor-Couette system, J. Fluid Mech. 590, 471 (2007).

[27] J. Abshagen, J. M. Lopez, F. Marques, and G. Pfister, Bursting dynamics due to a homoclinic cascade in Taylor-Couette flow, J. Fluid Mech. 613, 357 (2008). 
[28] J. M. Lopez, F. Marques, A. M. Rubio, and M. Avila, Crossflow instability of finite Bödewadt flows: Transients and spiral waves, Phys. Fluids 21, 114107 (2009).

[29] Y. Do, J. M. Lopez, and F. Marques, Optimal harmonic response in a confined Bödewadt boundary layer flow, Phys. Rev. E 82, 036301 (2010).

[30] S. Hughes and A. Randriamampianina, An improved projection scheme applied to pseudospectral methods for the incompressible Navier Stokes equations, Intnl J. Num. Meth. Fluids 28, 501 (1998).

[31] S. A. Orszag and A. T. Patera, Secondary instability of wallbounded shear flows, J. Fluid Mech. 128, 347 (1983).

[32] J. M. Lopez and J. Shen, An efficient spectral-projection method for the Navier-Stokes equations in cylindrical geometries I. Axisymmetric cases, J. Comput. Phys. 139, 308 (1998).

[33] J. M. Lopez, Numerical modeling of simple laboratory experiments of rotating flow, Ph.D. thesis, Applied Physics, Polytechnic University of Catalonia, Barcelona, 2015.

[34] M. Avila, M. Grimes, J. M. Lopez, and F. Marques, Global endwall effects on centrifugally stable flows, Phys. Fluids 20 , 104104 (2008)

[35] J. M. Lopez and F. Marques, Three-dimensional instabilities in a discretely heated annular flow: Onset of spatio-temporal complexity via defect dynamics, Phys. Fluids 26, 064102 (2014).

[36] F. Marques, I. Mercader, O. Batiste, and J. M. Lopez, Centrifugal effects in rotating convection: Axisymmetric states and threedimensional instabilities, J. Fluid Mech. 580, 303 (2007).

[37] J. M. Lopez, F. Marques, I. Mercader, and O. Batiste, Onset of convection in a moderate aspect-ratio rotating cylinder: Eckhaus-Benjamin-Feir instability, J. Fluid Mech. 590, 187 (2007).

[38] I. Mercader, A. Alonso, and O. Batiste, Spatio-temporal dynamics near the onset of convection for binary mixtures in cylindrical containers, Phys. Rev. E 77, 036302 (2008).

[39] J. M. Lopez and F. Marques, Rapidly rotating cylinder flow with an oscillating sidewall, Phys. Rev. E 89, 013019 (2014).
[40] F. Marques and J. M. Lopez, Spontaneous generation of a swirling plume in a stratified ambient, J. Fluid Mech. 761, 443 (2014).

[41] F. C. Moon, Chaotic and Fractal Dynamics: Introduction for Applied Scientists and Engineers (John Wiley \& Sons, New York, 2008).

[42] P. S. Marcus, Simulation of Taylor-Couette flow. Part 2. Numerical results for wavy-vortex flow with one traveling wave, J. Fluid Mech. 146, 65 (1984).

[43] A. Akonur and R. M. Lueptow, Three-dimensional velocity field for wavy Taylor-Couette flow, Phys. Fluids 15, 947 (2003).

[44] J. Abshagen and G. Pfister, Low-dimensional dynamics of axisymmetric modes in wavy Taylor vortex flow, in Physics of Rotating Fluids, Volume 549 of Lecture Notes in Physics, edited by C. Egbers and G. Pfister (Springer, New York, 2000), p. 84.

[45] H. Horner, Periodic perturbation on a period-doubling system, Phys. Rev. A 27, 1270 (1983).

[46] P. Bryant and K. Wiesenfeld, Suppression of period-doubling and nonlinear parametric effects in periodically perturbed systems, Phys. Rev. A 33, 2525 (1986).

[47] H. Svensmark and M. R. Samuelsen, Perturbed period-doubling bifurcation. I. Theory, Phys. Rev. B 41, 4181 (1990).

[48] C. Hoffmann, S. Altmeyer, A. Pinter, and M. Lcke, Transitions between Taylor vortices and spirals via wavy Taylor vortices and wavy spirals, New J. Phys. 11, 053002 (2009).

[49] S. Altmeyer, C. Hoffmann, M. Heise, J. Abshagen, A. Pinter, M. Lücke, and G. Pfister, End wall effects on the transitions between Taylor vortices and spiral vortices, Phys. Rev. E 81, 066313 (2010)

[50] A. S. Landsberg and E. Knobloch, Oscillatory bifurcation with broken translation symmetry, Phys. Rev. E 53, 3579 (1996).

[51] S. Viazzo and S. Poncet, Numerical simulation of the flow stability in a high aspect ratio Taylor-Couette system submitted to a radial temperature gradient, Computers \& Fluids 101, 15 (2014). 